\title{
Investigation on thermal protection and drag reduction by lateral jet in supersonic flows
}

\author{
Zhu Liang $^{1, \mathrm{a}}$, Chen Xiong ${ }^{1, \mathrm{~b}}$, Li Ying-kun ${ }^{1, \mathrm{c}}$, Yan Deng-chao ${ }^{1, \mathrm{~d}}$ \\ ${ }^{1}$ School of Mechanical Engineering, NJUST, Nanjing 210094, Jiangsu, China \\ a zhuliang203@126.com, b chenxiong@nust.edu.cn, ${ }^{c}$ lyk1007@126.com, dydc203@163.com
}

Key words: lateral jet; thermal protection system; drag; computational fluid dynamics

Abstract: A numerical code was developed to investigate the characteristics of drag and heat reduction by the lateral jet in supersonic flows, the Reynolds-average Navier-Stokes (RANS) equations was solved by using high resolution upwind scheme AUSMPW+, three order MUSCL reconstruction method and $\mathrm{k}-\omega \mathrm{SST}$ turbulence model with solving the unsteady heat transfer equations. The code was validated by an experimental case. The computed results indicate that: the numerical code can effectively capture the flow features, and the pressure and Stanton number on blunt body surface are significantly decreased with the addition of the lateral jet. The lateral jet was proved to be an effective method to reduce drag and heat.

\section{Introduction}

With the rapid developments of aerospace technology, supersonic aircraft has already got lots of attentions widely. The huge drag force and severe heating still present two major challenges for engineers. In order to make the supersonic aircraft has the long cruise performance, drag and heat reduction is of significant importance ${ }^{[1]}$. Flow control technology is usually applied to achieve the reduction of drag and heat. In general, spike, opposing jet, concentrated energy deposition and some other technologies has been widely studied ${ }^{[2,3,4]}$. The spike installed in front of vehicle changes the shape and the location of the shock wave to achieve the reduction of drag, but it is not effective to reduce the heat flux ${ }^{[5,6]}$. Especially the attack angle equals zero, the spike technology has good performance, but when the attack angle changes, the drag reduction performance becomes worse and brings about severe aerodynamic heating. This disadvantage limits the applications of spike to some extent. The opposing jet method was developed by Finley ${ }^{[7]}$ in 1966 and verified by experiment. The shock wave is pushed off the wall by the opposing jet, and the recirculation region is covered with cool gas. The wall heat flux keeps at low level because of the cool gas. On the other hand, the pressure along the wall decreases significantly because of the recirculation zone ${ }^{[8]}$. The basic flow structures and the physical mechanism of reduction of heat flux in opposing jet flow were analyzed by literature [9]. Recently, some novel combinatorial methods ${ }^{[10,11]}$ have been developed, including the combination of the opposing jet and the spike, the combination of the opposing jet and the energy deposition ,the combination of the opposing jet and the forward facing cavity ${ }^{[12,13]}$. Relevant researches indicate these strategies achieved effective reduction of drag and heat to some extent.

Jiangzonglin ${ }^{[14]}$ proposed a novel combination of the lateral jet and spike and verified this method by experiment. The results indicate this strategy has excellent performance in heat and drag reduction even the attack angle not equals zero.

In this paper the RANS equations are solved by finite volume method by using high resolution upwind scheme AUSMPW+, MUSCL reconstruction method and k- $\omega$ SST turbulence model with solving the unsteady heat transfer equations. The characteristics of drag and heat reduction by a combinational lateral jet and spike were numerical studied. 


\section{Governing equations and numerical method}

\section{Governing equations of fluid domain}

The two dimensional axisymmetric compressible Reynolds-Averaged Navier-Stokes (RANS) equations in differential form,

$$
\frac{\partial \boldsymbol{Q}}{\partial t}+\frac{\partial \boldsymbol{E}}{\partial x}+\frac{\partial \boldsymbol{F}}{\partial y}=\frac{\partial \boldsymbol{E}_{\boldsymbol{v}}}{\partial x}+\frac{\partial \boldsymbol{F}_{\boldsymbol{v}}}{\partial y}+\boldsymbol{H}+\boldsymbol{H}_{\boldsymbol{v}}
$$

Where $\boldsymbol{Q}$ is the vector of conservative variables; $\boldsymbol{E}, \boldsymbol{F}$ and $\boldsymbol{E}_{v}, \boldsymbol{F}_{v}$ are the convective and viscous parts of the flux vectors, respectively. $\boldsymbol{H}, \boldsymbol{H}_{v}$ are the convective and viscous axisymmetric source terms. The detailed description of these above vectors are as flowing,

$$
\begin{gathered}
\boldsymbol{Q}=\left[\begin{array}{l}
\rho \\
\rho u \\
\rho v \\
\rho E
\end{array}\right], \boldsymbol{E}=\left[\begin{array}{l}
\rho u \\
\rho u^{2}+p \\
\rho v u \\
(\rho E+p) u
\end{array}\right], \boldsymbol{F}=\left[\begin{array}{l}
\rho v \\
\rho u v \\
\rho v^{2}+p \\
(\rho E+p) v
\end{array}\right] \\
\boldsymbol{E}_{\boldsymbol{v}}=\left[\begin{array}{l}
0 \\
\tau_{x x} \\
\tau_{x y} \\
u \tau_{x x}+v \tau_{x y}+q_{x}
\end{array}\right], \boldsymbol{F}_{v}=\left[\begin{array}{l}
0 \\
\tau_{x y} \\
\tau_{y y} \\
u \tau_{x y}+v \tau_{y y}+q_{y}
\end{array}\right] \\
\boldsymbol{H}=-\frac{1}{y}\left[\begin{array}{l}
\rho v \\
\rho u v \\
\rho v^{2} \\
(\rho E+p) v
\end{array}\right], \boldsymbol{H}_{\boldsymbol{v}}=\frac{1}{y}\left[\begin{array}{l}
0 \\
\tau_{x y} \\
\tau_{y y}-\tau_{\theta \theta} \\
u \tau_{x y}+v \tau_{y y}+q_{y}
\end{array}\right]
\end{gathered}
$$

In these equations, $\rho, u$ and $v$ respectively stand for the density and velocity components; $p$ is the pressure.

Lots of existing researches indicate the flow field is in turbulent state, so k- $\omega$ shear stress transport turbulence model developed by Menter is applied here. This turbulence model uses blending functions to combine the advantages of $k-\varepsilon$ and $k-\omega$ and has been widely applied in engineering applications. Before conjugate heat transfer computation, assuming that the blunt body wall for adiabatic wall. Then the steady flow is calculated as the initial condition for conjugate heat transfer. All the numerical results based on the $t=0.5 \mathrm{~s}$.

\section{Governing equations of heat transfer}

The two-dimensional axisymmetric unsteady heat conduction equations is as following,

$$
\begin{gathered}
\rho_{s} C_{s} \frac{\partial T_{s}}{\partial t}=k_{s}\left(\frac{\partial^{2} T_{s}}{\partial x^{2}}+\frac{\partial^{2} T_{s}}{\partial y^{2}}\right)+H_{s} \\
H_{s}=\frac{1}{y} k_{s} \frac{\partial T_{s}}{\partial y}
\end{gathered}
$$

Where $\rho_{s}, \mathrm{C}_{\mathrm{s}}, \mathrm{T}_{\mathrm{s}}$ and $\mathrm{k}_{\mathrm{s}}$ represent the solid density, specific heat, temperature and thermal conductivity, respectively. $H_{\mathrm{s}}$ is the axisymmetric source terms.

\section{Numerical algorithm}

In order to capture the shock wave, expansion wave, shear layer and other complicated flow structures in the supersonic flows accurately, the three order MUSCL upwind scheme is applied here to discretize the convection terms, and a Van Albada limiter function is adopted to avoid the non-physical numerical oscillation. Then the convective fluxes through the cell face is computed by Advection Upstream Splitting Method by Pressure-Based Weight Function+ (AUSMPW+). For the viscous terms, the fluxes are computed by the standard central scheme. To improve the computation efficiency in unsteady flow case, a dual time stepping method is implemented for temporal discretization and the Lower-Upper Symmetric Gauss-Seidel (LU-SGS) implicit time method is 
applied in every time step.

A conjugate heat transfer method is adopted to describe the heat transfer between the fluid and solid domain. The temperature at the interface is obtained by ensuring the continuum of the heat flux density at the fluid-solid interface ${ }^{[15]}$. Figure 1 is the schematic of the conjugate heat transfer.

$$
-k_{\text {solid }} \cdot \frac{T_{b}-T_{s}}{\Delta n_{s}}=-k_{\text {fluid }} \cdot \frac{T_{f}-T_{b}}{\Delta n_{f}}
$$

The interface temperature $T_{b}$ can be obtained from the Eq. 5 , then $T_{b}$ is applied as boundary condition for fluid and solid domain, respectively. The RANS equations and heat transfer equations are solved by using the same time step.

The simulation model was chosen from the literature [9], shown in Fig 1. The diameter of the blunt body is $50 \mathrm{~mm}$, and the lateral jet width is $2 \mathrm{~mm}$ and the length $L_{0}$ of the spike is $20 \mathrm{~mm}$, other parameters can be found in table 1.

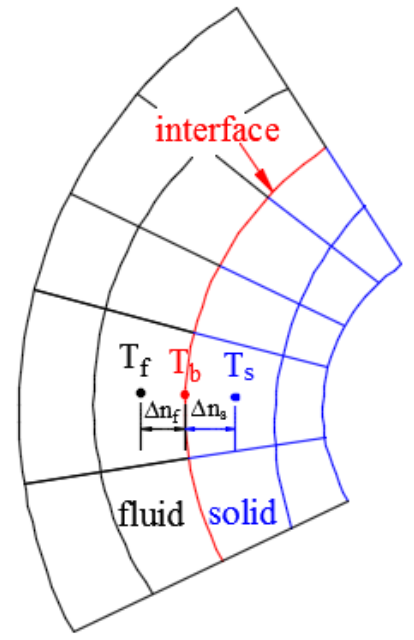

Fig 1. Schematic of the conjugate heat transfer

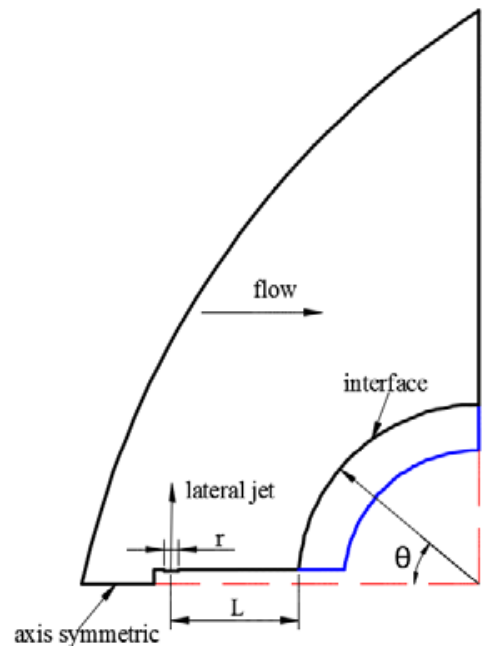

Fig 2. Computational model

In order to compute the wall heat flux accurately, the mesh near the wall was refined. PR is total pressure ratio and LR equals 0.9. The lateral jet Mach number is 1.0, and the total temperature of lateral jet is $300 K$.

$$
P R=\frac{P_{0 j}}{P_{0 \infty}}, L R=\frac{L}{L_{0}}
$$

Table 1 free stream conditions

\begin{tabular}{lccc}
\hline \multirow{2}{*}{ Free stream } & $M a$ & $P_{0 \infty}$ & $T_{0}$ \\
& 3.98 & $1.37 \mathrm{MPa}$ & $397 \mathrm{~K}$ \\
\hline
\end{tabular}

\section{Validation study}

In order to validate the present numerical code, a case chosen from literature [16] was simulated. Figure 3 give the comparison between the experimental data and the numerical results. It is clear that the numerical result agrees well with the experimental data, but there exists slight difference between them and the discrepancy may be induced by the turbulence model and the surface roughness. The comparison of the trends and the distributions indicate the numerical code in this paper can simulate the flow field and the wall heat flux. 


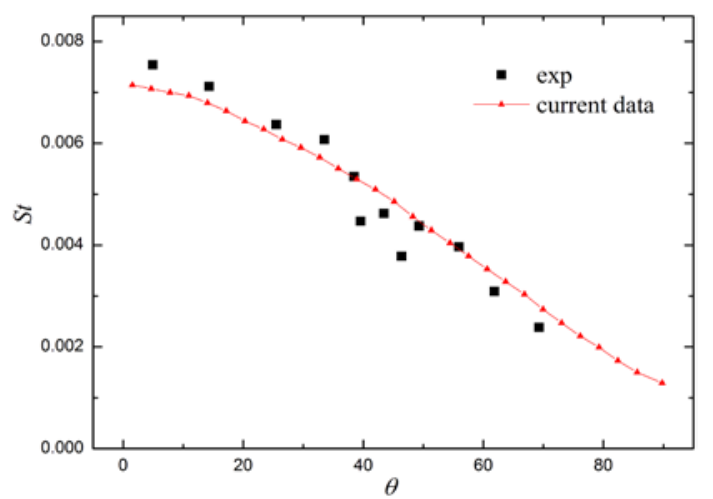

Fig 3. Comparison between experimental data and numerical results

\section{Numerical results and analysis}

To analyze the wall heat flux, the Stanton number is defined as

$$
\begin{gathered}
S t=\frac{q_{w}}{\left(T_{a w}-T_{w}\right) \rho_{\infty} c_{p} \mu_{\infty}} \\
T_{a w}=T_{\infty}\left\{1+[(\gamma-1) / 2] M a^{2} \operatorname{Pr}^{1 / 3}\right\}
\end{gathered}
$$

Where $q_{w}$ is the wall heat flux, $T_{\mathrm{aw}}$ is the adiabatic wall temperature, and $T_{\mathrm{w}}$ is the wall temperature. $M a, \rho_{\infty}$ and $T_{\infty}$ are represent the free stream Mach number, density, temperature. Pr is the Prandtl number.

Figure show the path line and Mach contour.

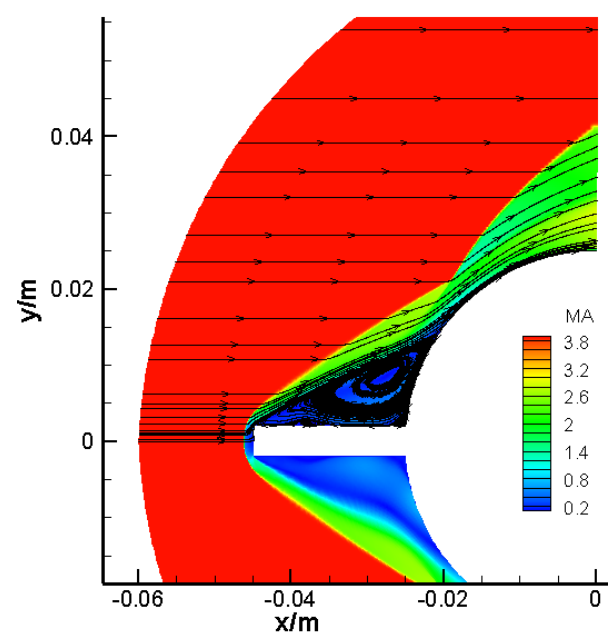

(a)

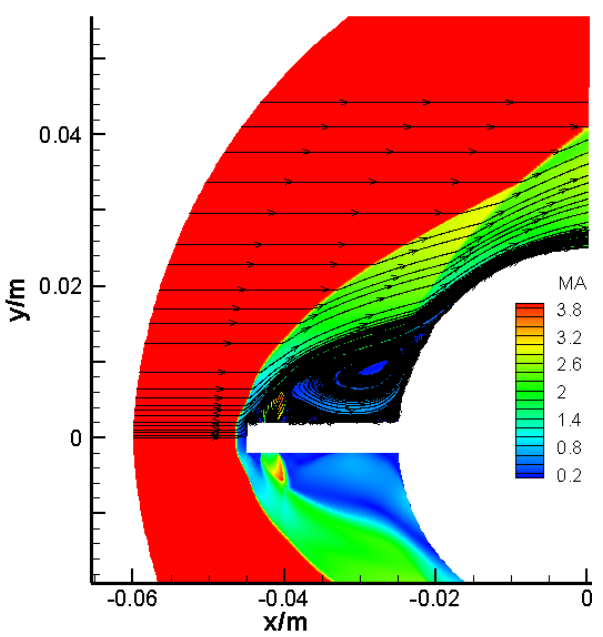

(b)

Fig. 4 streamline distribution and Mach contours (a) without lateral jet and (b) with lateral jet

In Fig. 4, it is obvious that the numerical results clearly captured the complex flow structure, such as the bow shock, the shear layer, the reattachment shock and the circulation zone and so on. The results also indicate that the numerical algorithm applied in this paper is suitable for supersonic complex jet flow field. From Fig (4) a, When the single spike is installed in head of the blunt body, the bow shock is formed in front of the spike and deflects to the blunt body and the bow shock gets closer to the wall. Finally, the bow shock hits the shoulder of the blunt body and is disturbed with the reattachment shock wave, and high heat flux is formed near the vicinity of the disturbed region. Relevant researches show that the shock-shock interaction near the wall brings about severe aerodynamic heating and limits the applications of spike.

Fig (4) b shows the reformed shock structure with lateral jet. The flow structure changes obviously as the lateral jet added to the spike. The jet gas expands after exit and the temperature decreases. From the Fig (4) b, it is clear the barrel shock is formed, and due to the free stream the barrel shock is deflective to the jet exit. Compared with the single spike, the conical shock is pushed 
away from the blunt body surface, and the recirculation zone beside the spike and in front of the blunt body becomes larger because of the lateral jet gas. This flow structure plays an important role in reduction of heat and drag. The larger recirculation zone leads to a larger conical shock angle, and the interaction of the conical shock with the reattachment shock could be relieved on the shoulder of the blunt body. In this case, the pressure and the temperature at the reattachment region are decreased significantly.

The comparison of the Stanton number of different cases is shown in Fig 5. Although the Stanton number at the fore part of the blunt body wall is decreased significantly, the peak Stanton number on the blunt body wall is about 0.018, which is much higher than the Stanton number without any measures. The high Stanton number is caused by the shock-shock interaction on the shoulder of the blunt body. When the lateral jet is applied, not only the peak Stanton number decreases to about 0.004, but also all the Stanton number along the blunt body wall becomes smaller than that without any measures. These comparisons of the Stanton number indicate the lateral jet is an effective way to reduce aerodynamic heating.

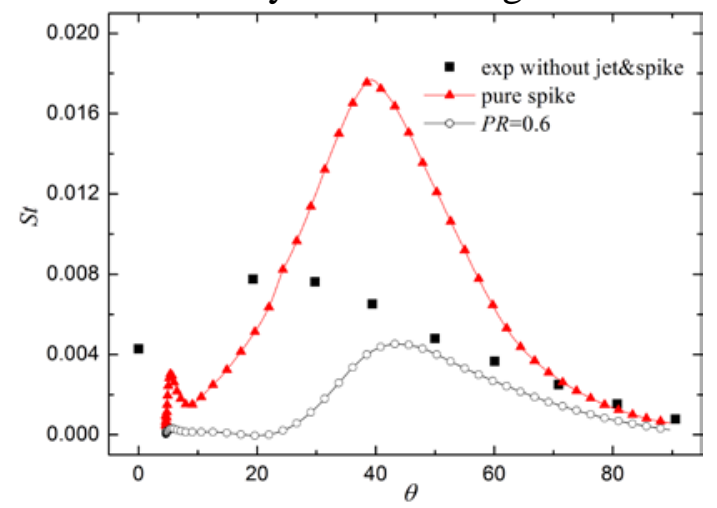

Fig. 5 Stanton number distribution of different cases

Figure 6 shows the comparison of pressure on the blunt body wall. When the single spike is applied, the pressure on the shoulder of the blunt body is little higher than that without any measures, but the pressure shows downward trend, while he pressure is decreased significantly as the lateral jet added.

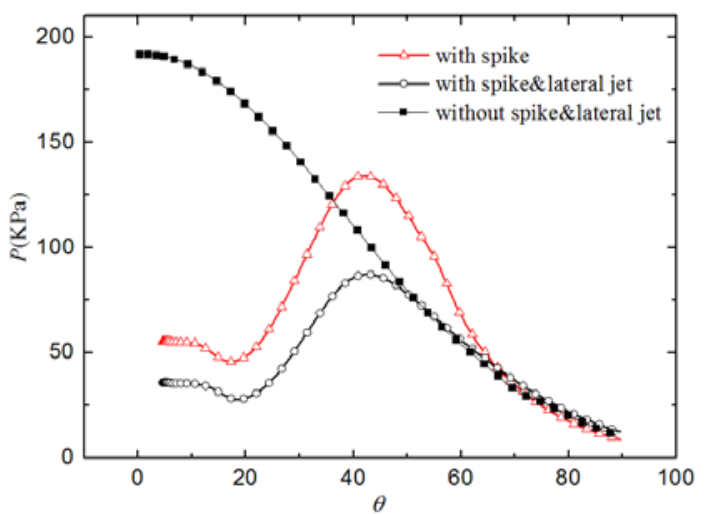

Fig. 6 Pressure distribution of different cases

\section{Summary}

A numerical code was developed to study the flow features and the heat transfer. From the above sections, the numerical investigations can be summarized as following. The numerical algorithm can effectively capture the flow features, and the conjugate heat transfer method is able to describe the heat transfer between the fluid and solid. Compare with the single spike, the addition of lateral jet plays an important role in reconstructing flow structure. From the numerical results, it is obvious the lateral jet is an effective method to reduce the drag and aerodynamic heating.

\section{References}

[1] Ho S Y, Paull A. Coupled thermal, structural and vibrational analysis of a hypersonic engine 
for flight test[J]. Aerospace Science \& Technology, 2006, 10(5):420-426.

[2] Huang W. A survey of drag and heat reduction in supersonic flows by a counterflowing jet and its combinations [J]. Journal of Zhejiang University-science A, 2015, 16(7):551-561.

[3] Ahmed M Y M, Qin N. Recent advances in the aerothermodynamics of spiked hypersonic vehicles [J]. Progress in Aerospace Sciences, 2011, 47(6):425-449.

[4] Kremeyer K, Sebastian K, Shu C W. Computational Study of Shock Mitigation and Drag Reduction by Pulsed Energy Lines [J]. Aiaa Journal, 2006, 44(8):1720-1731.

[5] Gerdroodbary M B, Imani M, Ganji D D. Heat reduction using conterflowing jet for a nose cone with aerodisk in hypersonic flow[J].Aerospace Science \& Technology, 2014, 39:652-665.

[6] Mansour K, Khorsandi M, Mansour K, et al. The drag reduction in spherical spiked blunt body[J]. Acta Astronautica, 2014, 99(8):92-98.

[7] Finley P J. The flow of a jet from a body opposing a supersonic free stream[J]. Journal of Fluid Mechanics, 1966, 26(26):337-368.

[8] Gerdroodbary M B, Bishehsari S, Hosseinalipour S M, et al. Transient analysis of counterflowing jet over highly blunt cone in hypersonic flow[J]. Acta Astronautica, 2012, 73(73):38-48.

[9] Hayashi K, Aso S, Tani Y. Numerical Study of Thermal Protection System by Opposing Jet[C]// Aiaa Aerospace Sciences Meeting and Exhibit. 2005.

[10] Gerdroodbary M B, Imani M, Ganji D D. Heat reduction using conterflowing jet for a nose cone with aerodisk in hypersonic flow[J]. Aerospace Science \& Technology, 2014, 39:652-665.

[11] Huang W, Liu J, Xia Z X. Drag reduction mechanism induced by a combinational opposing jet and spike concept in supersonic flows[J]. Acta Astronautica, 2015, 115:24-31.

[12] LU Hai-bo, LIU Wei-qiang. Investigation on Thermal Protection Efficiency of Hypersonic Vehicle Nose with Forward-Facing Cavity[J]. Journal of Astronautics, 2012, 33(8):1013-1018.

[13] Huang W, Yan L, Liu J, et al. Drag and heat reduction mechanism in the combinational opposing jet and acoustic cavity concept for hypersonic vehicles [J]. Aerospace Science \& Technology, 2015, 42:407-414.

[14] Yunfeng Liu. Zonglin Jiang. Concept of Non-ablative TPS for Hypersonic Vehicles[C]// Aiaa International Space Planes and Hypersonic Systems and Technologies Conference. 2011.

[15] CHEN Xiong, LI Ying-kun, LIU Rui et al. A Study of Thermal Protection Layer in Dual Pulse Motor Based on Conjugated Heat Transfer Method [J] Journal of Propulsion Technology, 2016, 37(1): 83-89. (in Chinese)

[16] Peery K M, Imlay S T. Blunt-body flow simulations[C]//Aiaa, Joint Propulsion Conference. 1988. 